\title{
Valoración de la severidad en enfermedades degenerativas articulares temporomandibulares mediante tomografía computarizada Cone Beam.
}

\section{Assessment of the severity of temporomandibular articular degenerative diseases through Cone Beam imaging.}

\author{
Mónica Firmani-Villarroel ${ }^{1 *}$, María Fernanda Cortés-Sylvester ${ }^{2}$, Cristian Burgos-lbarra $^{3}$
}

1. Cirujano Dentista, Magíster en Ciencias odontológicas con especialidad en Trastornos temporomandibulares y Dolor orofacial. Especialista en radiología Oral y maxilofacial. Profesora asistente. Departamento de Prótesis, Facultad de Odontología, Universidad de Chile. Chile. 2. Cirujano Dentista. Master en Trastornos temporomandibulares y Dolor orofacial. Especialista en radiología Oral y maxilofacial. Instructora Adjunta. Departamento de Patología y Medicina Oral, Facultad de Odontología, Universidad de Chile. Chile.

3. Cirujano Dentista. Especialista en Ortodoncia y Ortopedia maxilofacial. Complejo Asistencial Dr. Sótero del Río. Chile.

* Correspondencia Autor: Monica Firmani | Sergio Livingstone 943, Independencia, Santiago, Chile | E-mail:mfirmani@odontologia.uchile.cl.

Trabajo recibido el 20/12/2019

Trabajo revisado 26/04/2020

Aprobado para su publicación el 06/05/2020

\begin{abstract}
RESUMEN
Introducción: Muchos estudios han demostrado que las enfermedades degenerativas articulares Temporomandibulares (EDATM) provocan dolor, alteran la función modificando las estructuras esqueletales que se traducen en asimetrías faciales. La valoración imagenológica contribuye a un adecuado diagnóstico con el objetivo de optimizar la evaluación morfológica de las articulaciones temporomandibulares. Metodología: Se realizó una búsqueda electrónica en las bases de datos de PubMed, Google Scholar y SciELO. La estrategia de búsqueda se realizó utilizando una combinación de términos con el objetivo de analizar la valoración de las características imagenológicas y de volumen condilar. Resultados y Discusión: De un total de 9807 artículos se seleccionaron 18 que cumplían con los requisitos. Se han propuesto muchas categorías para clasificar la severidad imagenológica de la EDATM sumado al advenimiento de softwares y reconstrucciones tridimensionales que han propuesto categorías a través de algoritmos matemáticos y de superposición de imagen que son un gran aporte para el diagnóstico, la toma decisiones en la elección del plan de tratamiento y en el seguimiento. Conclusiones: La valoración de la severidad de las EDATM son claves para que la investigación clínica permita esclarecer los procesos que se relacionan con el objeto de valorar la progresión de esta enfermedad.
\end{abstract}

PALABRAS CLAVE:

Enfermedad degenerativa articular temporomandibular; Tomografía computarizada Cone Beam; Volumen condilar.

Int. J. Inter. Dent Vol. 14(1); 37-43, 2021.

\section{ABSTRACT}

Introduction: Many studies have shown that Temporomandibular degenerative joint diseases (TMDJD) cause pain, alter function by modifying skeletal structures that result in facial asymmetries. Imaging evaluation contributes to an adequate diagnosis with the aim of optimizing the morphological evaluation of the temporomandibular joints. Methodology: An electronic search was performed in the PubMed, Google Scholar and SciELO databases. The search strategy was performed using a combination of terms in order to analyze the assessment of imaging characteristics and condylar volume. Results and Discussion: From a total of 9807 articles, 18 were selected that met the requirements. Many categories have been proposed to classify the imaging severity of the TMDJD added to the advent of software and three-dimensional reconstructions that have proposed categories through mathematical algorithms and image superposition that are a great contribution to diagnosis, decision-making and choice of the treatment plan and follow-up. Conclusions: The assessment of the severity of TMDJD is key for clinical research in order to clarify the processes that are related to assessing the progression of this disease.

KEY WORDS:

Temporomandibular degenerative joint disease; Cone Beam; Condylar volume.

Int. J. Inter. Dent Vol. 14(1); 37-43, 2021.

\section{INTRODUCCIÓN:}

La mandíbula se articula con el cráneo tanto a través de la relación de los dientes antagonistas en oclusión como por las articulaciones temporomandibulares (ATMs), su interrelación durante el crecimiento es evidente. Una alteración en las ATMs en esta etapa afecta la relación de contacto dentario y el patrón de crecimiento mandibular puede ir cambiando, favoreciendo asimetrías esqueletales del complejo craneofacial y su función ${ }^{(1)}$.

La ATM es una articulación sinovial con características propias en el 
organismo. Conocer su anatomía durante el crecimiento y desarrollo y su biomecánica es fundamental para la evaluación imagenológica, puesto que la apariencia en la imagen representa un punto de un proceso continuo en el crecimiento y desarrollo, así como en el proceso de una enfermedad ${ }^{(2)}$.

El cóndilo mandibular está cubierto por una capa de fibrocartílago separada por una fina capa proliferativa de la superficie de cartílago maduro e hipertrófico de las capas inferiores ${ }^{(3)}$. La capa de fibrocartílago contiene predominantemente fibroblastos y colágeno tipo I, células mesenquimáticas indiferenciadas que se encuentran en la zona proliferativa, sirviendo como reservorio celular. La zona inferior es constituida por condrocitos diferenciados y colágeno de tipo I y II. Desde el punto de vista embriológico, el cartílago de las ATMs se constituye como de tipo secundario formado por endostio y periostio, mientras que el cartílago que es de tipo primario es el que precede la formación ósea(3).

Si producto de traumatismos o de algún proceso patológico en las ATMs en etapa de desarrollo se daña la capa proliferativa, esto determina una alteración en el crecimiento y desarrollo esqueletal, resultando en asimetrías faciales transversales en el caso de que sea unilateral o en el sentido sagital si son bilaterales. Si hay un cambio en la cantidad de tejido proliferativo presente, éste puede causar un cambio en el patrón de desarrollo, tal como una hipoplasia, hiperplasia o microsomía hemifacial que son considerados trastornos de desarrollo(2).

Dentro de los trastornos adquiridos, la osteoartritis (OA) también conocida como enfermedad degenerativa articular (EDA), es uno de los trastornos más comunes que afectan las ATMs. Su etiología es compleja y multifactorial. Se ha demostrado que la OA temporomandibular (OATM) se produce producto de una inflamación local que ocurre cuando se rompe el equilibrio entre la destrucción y reparación de los tejidos articulares ${ }^{(4)} \mathrm{La}$ OA primaria y la OA sintomática o secundaria han sido descritas, la última se produce cuando cargas extrínsecas e intrínsecas exceden la capacidad adaptativa de los tejidos frente a la demanda funcional o a parafuncionales, secundaria a desplazamientos discales, trauma, sobrecarga funcional ${ }^{(5)}$. Esto genera una esclerosis subcondral, una baja resistencia articular y destrucción del fibrocartílago y al hueso subcondral. Dentro de los factores asociados están la inmovilización articular, los cambios oclusales, entre otros las alteraciones fisiológicas, hormonales y psicológicas han sido descritas ${ }^{(6)}$.

La osteoartritis y la osteoartrosis se enmarcan en las enfermedades degenerativas articulares, clasificados por el consorcio internacional de DC/TMD y la AAOP (American Association of Orofacial Pain) dentro de la taxonomía expandida para los trastornos temporomandibulares eje I, del capítulo de las enfermedades articulares ${ }^{(7)}$.

La osteoartrosis se diferencia de la osteoartritis, en que esta última cursa con dolor, sin embargo, estos términos no han sido usados consistentemente en medicina, por lo tanto, se prefiere subclasificarlos dentro de las enfermedades degenerativas articulares temporomandibulares (EDATM). Cuando la EDATM ocurre con dolor, se adjunta el diagnóstico de artralgia, así como también el diagnóstico de desplazamiento discal en el caso de que así ocurra. Para realizar el diagnóstico el consenso señala que, tiene que haber una historia consistente de ruidos articulares, de al menos de 30 días de evolución, de tipo crépito durante la función, o en el movimiento articular durante el examen. Los autores señalan que el algoritmo diagnóstico tiene una baja sensibilidad en las ATMs, por lo tanto, el diagnóstico clínico inicial queda sujeto a confirmación por medio de la imagen; de resonancia magnética para casos de desplazamientos discales y de tomografía computarizada helicoidal multicorte (TC) o de tomografía computarizada de haz cónico o Cone Beam Computer tomography (Según sus siglas en inglés "CBCT"), en caso de $\operatorname{EDATM}^{(7)}$. Para valorar la imagen ósea de las ATMs se indica preferir la tomografía computarizada, se recomienda protocolizar las imágenes axialmente corregidas a lo largo del eje mayor del cóndilo mandibular. La CBTC posee un archivo visualizador DICOM que permite posicionar correctamente las estructuras en los planos sagital oblicua y coronal oblicuo ${ }^{(2,8)}$.

El diagnóstico en la TC debe ser positivo al menos en uno de los siguientes criterios imagenológicos, cuya terminología debe ser consistente para minimizar el sesgo entre los radiólogos; la presencia de erosiones de la cortical del cóndilo mandibular (Figura 1e); de quistes subcondrales (Figura 1f); son signos que corresponden realmente a degeneración ósea subcondral. Cuando observamos esclerosis generalizada (Figura 1d) y osteofitos (Figura 1b), aunque sean pequeños, son signos que indican degradación del cartílago y están asociados a la progresión de la EDATM. El aplanamiento (Figura 1c), la esclerosis subcortical y el estrechamiento del espacio articular son considerados hallazgos no concluyentes, constituyéndose como hallazgos inespecíficos. Puesto que pueden representar una variación normal, de envejecimiento y/o remodelación o bien, ser la imagen precursora de una franca EDATM, situación que no es predecible mediante la imagen de TC/CBCT. Los cuerpos libres dentro de la articulación no son considerados como signos de enfermedad degenerativa articular, a menos que haya otros signos presentes ${ }^{(7,9,10,11)}$.

Ahmad y $\operatorname{cols}^{(9)}$, en el año 2009 publican como parte del proyecto multicéntrico de validación del RDC/TMD, criterios imagenológicos diagnósticos para ATM utilizando radiografía panorámica, resonancia magnética (RM) y TC, siendo esta última considerada como Goldstandard para evaluación superficies óseas articulares ${ }^{(9)}$. Para este sistema de clasificación diagnóstica se utilizó el término de OATM, aunque indican que el término EDATM sería más adecuado para la interpretación de imágenes y radiografías cuando no existe información clínica disponible. Este sistema propuesto para OATM diagnostica las estructuras articulares óseas basándose en la evaluación de presencia de erosión, pseudoquistes subcorticales, osteofitos y esclerosis generalizada en tres categorías. Grupo A que corresponde a que no presenta la enfermedad, grupo B que corresponde a que presenta características indeterminadas o inespecíficas y grupo $\mathrm{C}$ que corresponde a la presencia de $\operatorname{EDATM}^{(9)}$.

Para esta clasificación, los autores excluyeron la evaluación del espacio articular, la esclerosis subcortical localizada, el aplanamiento de las superficies articulares y la presencia de cuerpos libres intraarticulares, dada la existencia de evidencia que indica que estos signos poseerían un valor predictor indeterminado, por otro lado, esta clasificación no permite evaluar diferentes grados de severidad, sólo discrimina entre ausencia, signos indeterminados o presencia de la enfermedad, lo que representa una limitación en el estudio de esta patología ${ }^{(9)}$. Sobre esta clasificación, el test diagnóstico utilizado fue la TC. Sin embargo, Larheim y cols(8), en una revisión sobre diagnóstico de ATM mediante Cone Beam, concluyen que tanto la TC como la CBCT son exámenes confiables en el diagnóstico de cambios degenerativos articulares y que la precisión diagnóstica de ambos es similar para patología degenerativa ósea. No obstante, el beneficio de exponer al paciente a una menor radiación en el caso de usar Cone Beam lo hace el examen de elección ${ }^{(8,12)}$. En 2018 Hilgenberg y cols ${ }^{(13)}$ suscriben estas afirmaciones y además agregan que el Cone Beam debe ser de elección para evaluar la progresión de la enfermedad en el tiempo.

Los criterios antes expuestos evitaban señalar la severidad o la extensión de la enfermedad. Aunque las designaciones como leve, moderada y severa son útiles para la clínica, como las propuestas por Tanaka $2008^{(14)}$ y Koos $2013^{(15)}$, son frecuentemente subjetivas y difíciles de estandarizar entre las interpretaciones.

\section{METODOLOGÍA:}

Se realizó una búsqueda electrónica en las bases de datos de PubMed, Google Scholar y SciELO. La estrategia de búsqueda se realizó utilizando la siguiente combinación de conectores booleanos y términos

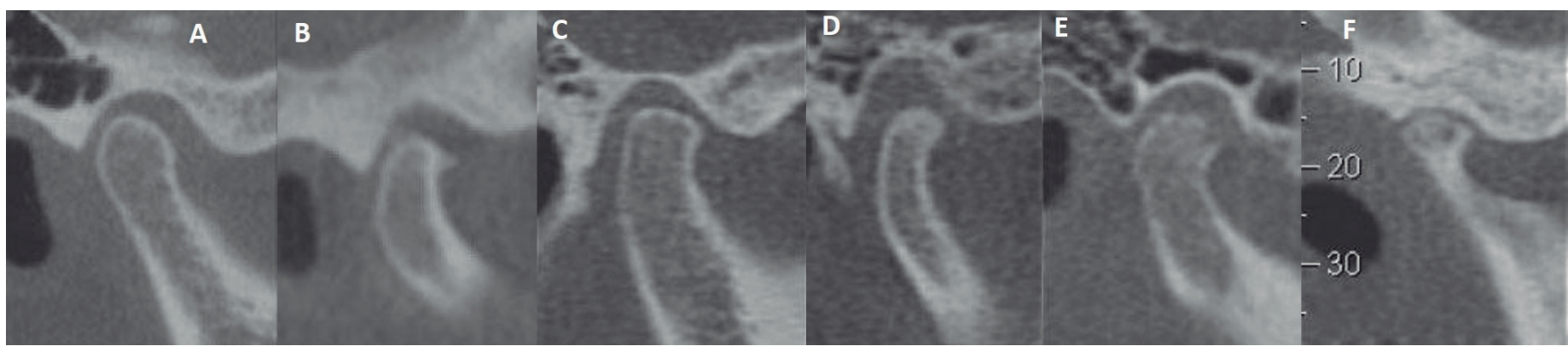

Figura 1. Cortes sagitales oblicuos de ATM en máxima intercuspidación (MIC). La morfología del cóndilo se clasificó en A) sin cambios óseos; B) osetofito; C) aplanamiento; D) esclerosis; E) erosión y F) pseuodquiste. Imagen tomada de Dos Anjos Pontual ML, Freire JSL, Barbosa JMN, Frazão MAG, Dos Anjos Pontual A. Evaluation of bone changes in the temporomandibular joint using cone beam CT. Dentomaxillofacial Radiol. 2012;41:24-29(10). 
MeSh: ((Temporomandibular diseases) OR (mandibular Condyle) AND (osteoarthritis) OR (degenerative joint disease) AND (diagnostic imaging) OR (three dimensional imaging) OR (cone beam computed tomography)). Se complementa con una búsqueda electrónica realizando una búsqueda manual selectiva de las referencias de los artículos previamente incluidos. Luego de eliminar los duplicados, se verificó la relevancia de los artículos evaluando el título y resumen. Cuando existió dudas de la relevancia se procedió a la lectura del artículo completo.

De un total de 9807 artículos, se seleccionaron 18 que cumplían con los requisitos de valoración de las características imagenológicas y de volumen condilar con el objetivo de optimizar el valor diagnóstico imagenológico que nos permita valorar la progresión de la enfermedad y su variabilidad en el tiempo.

\section{RESULTADOS Y DISCUSIÓN:}

De un total de 9807 artículos, se seleccionaron 18 que cumplían con los requisitos de valoración de las características imagenológicas y de volumen condilar con el objetivo de optimizar el valor diagnóstico imagenológico que nos permita valorar la progresión de la enfermedad y su variabilidad en el tiempo. (ver Tabla 1).

La EDATM se ha descrito como una enfermedad progresiva cuya patogénesis subyacente no está esclarecida en su complejidad. El hecho que sea progresiva desafía su valoración y la elección del plan de tratamiento, puesto que involucra tanto cambios adaptativos, caracterizados por cambios remodelativos como cambios regresivos caracterizados por reabsorciones óseas agresivas, deformando las estructuras y causando deficiencias funcionales ${ }^{(16)}$.

La mayoría de los criterios diagnósticos en EDATM se basaban en señalar los cambios patológicos en las ATMs. Uemura y cols(17) en 1979, describen los conceptos de erosión, osteofitos, proliferación marginal, deformación, aplanamiento, concavidad, eburnación y osteoesclerosis observados en radiografía convencional, conceptos que son usados universalmente. En 2007 Koyama y cols ${ }^{(12)}$ publican una clasificación propia a partir de imágenes en tomografía computarizada de la región temporomandibular, a partir de los cortes axiales y sagitales. Clasificaron los cambios óseos que se limitan al contorno de la superficie condilar en 5 tipos: N, sin cambios óseos; F, aplanamiento del contorno; E, erosión con o sin irregularidad de la cortical; $D$, deformidad con apariencia de pico de pájaro; S, deformidad acompañado de erosión con y sin irregularidad de la cortical. Esta clasificación fue propuesta con el objetivo de evaluar la progresión de la enfermedad. Evaluaron 102 articulaciones en su estudio, observaron que los cambios resortivos óseos varían a cambios resortivos con esclerosis y luego pasan a cambios escleróticos. Los aplanamientos $(F)$ y erosiones $(E)$ son etapas iniciales, ellos confirmaron que puede en algunos casos tener una evolución regresiva a sin cambios (N). La transición de la erosión (E) a aplanamiento $(F)$ y viceversa también fue observada en su estudio. Además, ellos mostraron que la erosión ( $E$ ) avanza a deformación (D) y a deformación acompañada de erosión (S) debido a la progresión de la resorción condilar. Sin embargo observaron que hay casos en que el tipo de deformación acompañada de erosión (S), pasa a volverse solo a deformación (D), como resultado de la restauración de la superficie cortical.

En 2014, Schiffman y cols ${ }^{(7)}$ publican la nueva actualización de criterios diagnósticos para los Trastornos temporomandibulares DC/TMD donde categorizan las distintas patologías musculoesqueléticas de la región. Posteriormente en el año 2016 Ahmad y Schiffman ${ }^{(18)}$ indican que para evaluar la articulación temporomandibular es necesario describir la extensión de la enfermedad, puesto que permite determinar la estabilidad o progresión de esta, describiendo una graduación que puede ser utilizada para su interpretación tanto en la TC, CBCT como RM. Los autores proponen una clasificación donde la EDATM está en Grado 1 si están presentes las siguientes características: un osteofito de longitud < a $2 \mathrm{~mm}$ visto en un corte sagital oblicuo, una erosión única de una longitud $<$ a 2 $\mathrm{mm}$ o un pseudo quiste único < a $2 \mathrm{~mm}$. Y como Grado 2 se observaría: un osteofito de longitud $\geq$ a $2 \mathrm{~mm}$, visto en un corte sagital oblicuo, una erosión única de una longitud $\geq$ a $2 \mathrm{~mm}$ en ancho y profundidad, o múltiples en cualquier tamaño, o un pseudo quiste único $\geq a 2 \mathrm{~mm}$, o más de uno en cualquier tamaño, o bien dos o más signos imagenológicos correspondientes a grado 1. Los autores señalan la importancia de que los hallazgos sean visibles en dos vistas tanto sagitales como coronales, además señalan que, si la erosión y el pseudoquiste aparecen como continuas, debe considerarse como erosión.

Por otro lado, Tamimi y cols ${ }^{(2)}$, indican la necesidad de evaluar la altura de los contornos óseos del cóndilo mandibular para determinar la posible pérdida de altura condilar o de tejido óseo articular. Esto debe evaluarse en una vista sagital oblicua de la ATM. En dicho corte se visualiza donde la cortical posterior del cuello del cóndilo se estrecha hasta un punto de la máxima convexidad posterior de éste, tras lo cual la cortical que cubre a la cabeza del cóndilo se vuelve muy fina en relación a la superficie articular hasta descender hacia la cortical anterior justo sobre la fosita pterigoidea, en donde la cortical comienza a engrosarse caudalmente en la superficie anterior del cuello del cóndilo. En un cóndilo normal el punto cortical posterior es más bajo que el anterior y la distancia entre ambas alturas es mayor conforme avanza el desarrollo hasta un cóndilo adulto. En una vista coronal oblicua, los contornos corticales medial y lateral se pueden conectar con una línea que pasa entre ambos, al igual que en la vista sagital oblicua como si fuese el ecuador del cóndilo, debiendo existir bastante masa ósea uniforme por sobre este ecuador. Una pérdida de hueso en la superficie articular pudiese indicar cambios degenerativos o inflamatorios y resulta en una migración craneal de los contornos corticales en relación a la fosa mandibular, lo que pudiese ser clave en diferenciar hipoplasia de cóndilo y EDATM.

El estudio del volumen condilar ha captado el interés científico por décadas, puesto que la cuantificación tridimensional es crítica para comparar la capacidad de las diferentes modalidades terapéuticas en detener la enfermedad ${ }^{(4)}$. Cevidanes y cols ${ }^{(19)}$, en 2009 muestran un estudio piloto con 20 pacientes, donde hicieron una valoración de los cambios patológicos en articulaciones con trastornos degenerativos en imágenes virtuales 3D y concluyen que los cambios patológicos son frecuentes. La valoración virtual $3 \mathrm{D}$ muestra hasta los pequeños cambios que pueden ser factores indicativos del inicio de la remodelación en estos pacientes. Los autores proponen una clasificación de remodelación degenerativa descrita como leve, moderada y severa para las distintas características de la enfermedad, como aplanamiento, erosiones, y osteofitos que pueden visualizarse en imágenes virtuales ${ }^{(19)}$. (Figura 2 )

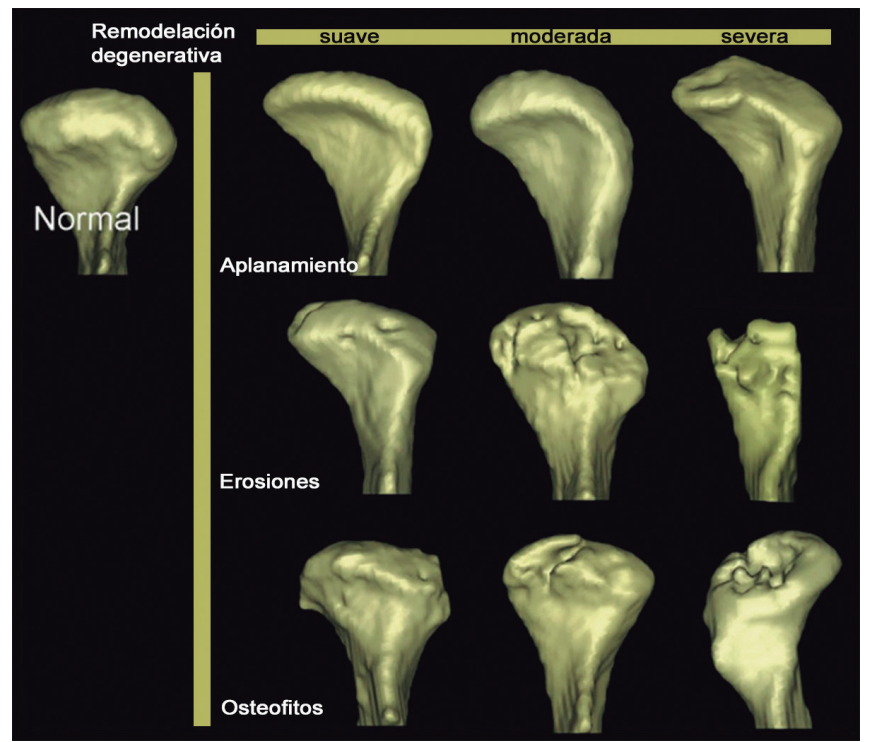

Figura 2. Se muestra la asociación de la forma condilar asociada a los cambios. el eje vertical describe progresión (aplanamiento, erosiones y osteofitos) de los cambios degenerativos, mientras que el eje horizontal representa la severidad. Imagen tomada de Cevidanes LH, Walker DG, Styner M, Lim PF. Condylar Resorption in Patients With Tmd. Craniofacial growth Ser. 2009; 46:147-157(19).

Paniagua y cols $^{(20)}$ en 2011 realizan un estudio para validar una metodología SPHARM-PDM de evaluación volumétrica condilar en CBCT y lo aplicaron a un sujeto pre y postratamiento quirúrgico, señalando que la limitación de los estudios volumétricos está en no detectar defectos ni cambios estructurales en el espesor del tejido, sin embargo reflejan el tamaño global de las estructuras y permiten localizar y cuantificar los cambios morfológicos entre estructuras sanas y patológicas, constituyéndose una herramienta para valorar efectos terapéuticos y permitiendo así realizar investigaciones entre cambios morfológicos y marcadores tanto clínicos como biológicos.

Schilling y $\operatorname{cols}^{(4)}$ en 2014, investigaron la confiabilidad de los registros tridimensionales por medio de la superposición regional en tres dimensiones de las estructuras temporomandibulares en sujetos en el tiempo, usando volúmenes a partir de imágenes de СВСТ en 12 pacientes sanos y 12 pacientes con EDATM. Los exámenes se tomaron pre operativamente, inmediatamente post cirugía y después de 1 año de seguimiento del tratamiento quirúrgico mandibular, utilizando puntos de 
referencia anatómicos y por correspondencia computacional por medio de softwares SPHARM-PDM. Concluyen que el hueso de los cóndilos al estar justo bajo el fibrocartílago, lo que lo hace particularmente susceptible a la presencia de inflamación, predispone a que esta estructura esté sujeta a cambios dinámicos en su morfología, lo que la hace especialmente vulnerable a cambios no necesariamente secundarios a la degradación del fibrocartílago. Además, sostienen que, aunque las diferencias entre los observadores calibrados existen, estas son mínimas, siendo la valoración tridimensional una herramienta confiable.

Kim y cols $^{(21)}$ en 2016, realizan un estudio donde analizan la asimetría volumétrica de las estructuras temporomandibulares. Los autores comparan un grupo de pacientes con asimetría con un grupo control, según los resultados de análisis de asimetría de Habets y col. (22). El estudio volumétrico lo realizan a partir de imágenes DICOM y utilizando el Software Simplant OMS 13.0, determinando puntos específicos tanto para el proceso condilar como para la fosa mandibular. Los autores concluyen que la asimetría facial no solo compromete el proceso condilar, sino que también la fosa mandibular, y que las diferencias entre las estructuras articulares en pacientes asimétricos se da predominantemente por un cóndilo más pequeño.

Gomes y cols en 2015, evaluaron la morfología condilar a través del software "The 3-D shape correspondence analysis (SPHARM-PDM)" y clasificaron la morfología condilar a través de puntos de referencia anatómicos. Proponen un sistema de clasificación que llamaron Dendograma, a partir de la jerarquización de las diferencias entre todos los sujetos sanos, comparados con aquellos con una larga historia de signos y síntomas de osteoartritis, identificando 6 subgrupos que, con la imagen superpuesta del cóndilo sano, se puede ver en imágenes las grandes diferencias que presentan. Jerarquizan las distintas morfologías de acuerdo al volumen y la forma condilares en 6 bloques. En el 6 se encuentran los cóndilos sanos, y se evidencia la progresión de la enfermedad hasta el bloque 3 , en el bloque 1 están los cóndilos más afectados, aquellos más pequeños y con grandes erosiones y por último en el bloque 2 se encuentran aquellos deformados con cambios proliferativos. Los autores destacan la importancia de asociar estos hallazgos con biomarcadores de destrucción tisular, para poder establecer un diagnóstico más acertado(16).

El grupo de estudio de Cevidanes y cols en $2015^{(23)}$, por medio de los mismos sistemas de superposición de estructuras y por medio de los softwares antes descritos, proponen un índice de severidad de EDATM, estableciendo 5 categorías, una que llamaron de crecimiento excesivo, por estar sobre el promedio del volumen de los cóndilos sanos, y 4 categorías de degeneración; las denomina como leve, moderado, marcado y severo. Señalan también que hay una amplia variabilidad de las formas en los pacientes con EDATM, siendo la más predominante el aplanamiento del polo lateral del cóndilo.

Por otro lado, Gumussoy y cols ${ }^{(24)}$ en un estudio reciente, realizan un análisis tridimensional óseo microestructural en pacientes con EDATM, a partir de 147 imágenes de ATMs de 88 individuos. Para analizar los cóndilos, se clasificó cada cóndilo en 1 a 3 grupos según el método de Muir y Goss ${ }^{(25)}$ del año 1990; el Grupo 0 se considera a los cóndilos normal-control; al Grupo 1, asociado a cambios erosivos osteoartrósicos moderados y al Grupo 2, incluyeron los cambios erosivos osteoartrósicos severos, tras lo que calcularon la dimensión fractal del cóndilo a partir de imágenes binarias utilizando SkyScan ${ }^{\mathrm{T} M}$ software. Este estudio preliminar concluye que el análisis fractal pudiese ser una herramienta valiosa para potenciar la capacidad diagnóstica del CBCT en la evaluación de EDATM, sin embargo, utilizaron una clasificación bidimensional y que se enfoca principalmente en cambios erosivos.

Los autores Ribera, Dumast y Cols ${ }^{(26)}$ en 2019 publican lo que llaman "Analizador de variación de la forma", (SVA de acuerdo a sus siglas en inglés), que permite realizar un estadiaje de los cambios morfológicos de la EDATM. Los autores realizan una clasificación, Codifican 6 grupos, basándose en la combinación de rasgos geométricos, descriptores de forma y temperatura de Kernel, posteriormente lo compararon con 9 algoritmos diferentes, utilizando el test de trabajo de red neuronal, que fue entrenado para clasificar en una matriz dimensionada, para que busque e identifique patrones discriminatorios entre los rasgos codificados, para mejorar la confiabilidad de la clasificación. Describen al Grupo 0 como sano, seleccionado como grupo control; el grupo 1 como cercano a lo sano; el grupo 2 como degeneración 1; al grupo 3 como degeneración 2; al grupo 4 como degeneración 3; al grupo 5 como degeneración 4 y 5 (Figura 3). Los autores señalan que esta clasificación les parece prometedora puesto que permite evaluar la forma condilar del paciente a lo largo de su enfermedad de manera más precisa. El repositorio está disponible en https://github.com/DCBIA-OrthoLab/ShapeVariationAnalyzer. Señalan que su propuesta debe incluir muestras de otros biomarcadores de la enfermedad para que esta red de trabajo tenga más fuerza.

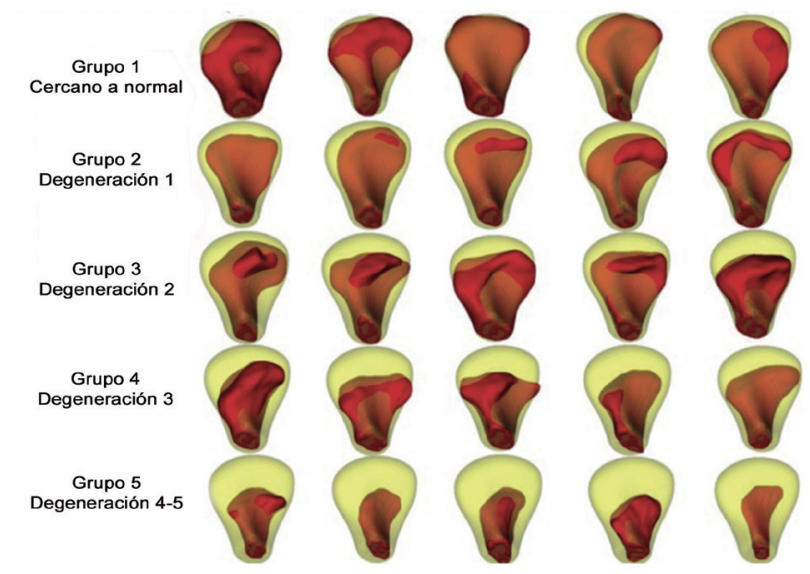

Figura 3. Se muestra en amarillo el promedio de volumen del grupo control y en Rojo el volumen superpuesto de la graduación de la pérdida de volumen condilar y su clasificación: G1: cercado a lo sano, G2: Degeneración 1, G3: Degeneración 2, G4: Degeneración 3 y G5: Degeneración 4-5. Imagen tomada de Ribera NT, de Dumast P, Yatabe $M$, et al. Shape variation analyzer: a classifier for temporomandibular joint damaged by osteoarthritis. Proc SPIE Int Soc Opt Eng. 2019; 10950:1095021(26).

\section{CONCLUSIÓN:}

Las Articulaciones temporomandibulares están sujetas a transformaciones morfológicas a lo largo de la vida de los individuos, las cuales no todas son manifestaciones de una enfermedad degenerativa articular, sin embargo, hay rasgos característicos que sí lo son e identificarlos de manera temprana es fundamental para elegir terapias que ayuden a la calidad de vida de los pacientes. Ayudando a establecer aproximaciones terapéuticas que sean confiables a través de técnicas de valoración de la forma y tamaño condilares en tres dimensiones o por superposición volumétricas, lo que permitirá entender mejor los mecanismos de la enfermedad y sopesar la efectividad de los diferentes protocolos terapéuticos.

\section{CONFLICTO DE INTERÉS}

Los autores no declaran conflicto de interés.

Tabla 1. Resumen descriptivo de los papers seleccionados $(n=18)$

\begin{tabular}{|c|c|c|c|c|}
\hline Autor y añ & & Objetivo & Metodología & Conclusiones \\
\hline $\begin{array}{l}\text { Habets } \\
\text { cols, } 1988\end{array}$ & $y$ & $\begin{array}{l}\text { Evaluar asimetría en pacientes con } \\
\text { signos y síntomas de TTM, observados } \\
\text { en una radiografía panorámica }\end{array}$ & $\begin{array}{l}\text { Compararon mediciones verticales } \\
\text { de cóndilo y rama de } 92 \text { pacientes } \\
\text { asintomáticos y } 60 \text { sintomáticos, } \\
\text { calculando la simetría entre ambos lados }\end{array}$ & $\begin{array}{l}\text { Observaron que la atura del cóndilo } \\
\text { era menor en pacientes sintomáticos } \\
\text { así como mayor presencia de asimetría } \\
\text { intra sujeto en pacientes en estos } \\
\text { pacientes comparados con los controles. } \\
\text { Concluyen que observar asimetrías en } \\
\text { radiografías panorámicas puede ayudar } \\
\text { a distinguir en el paciente el riesgo de } \\
\text { desarrollar TTM }\end{array}$ \\
\hline
\end{tabular}


Tabla 1. Resumen descriptivo de los papers seleccionados $(n=18)$ (continuación)

\begin{tabular}{lll}
\hline Autor y año & \multicolumn{1}{c}{ Objetivo } & \multicolumn{1}{c}{ Metodología } \\
\hline K o y a m a , Evaluación y seguimiento de incidencia & Se evaluaron 516 sujetos, 51 de ellos con \\
Is h i y a m a & y cambios óseos condilares en paciente & seguimiento que se realizó entre los 3 y \\
y Hayashi, con TTM observados en TC & 18 meses. Se desarrollaron criterios de \\
2007 & evaluación; tipo $\mathrm{N}$ - sin cambios óseos, \\
& tipo $\mathrm{F}$ - aplanamiento de superficies, \\
& tipo E - erosión superficial, tipo D - \\
& deformación, tipo S -deformación con \\
& erosión.
\end{tabular}

Cevidanes y Determinar las diferencias morfológicas cols, 2009 entre articulaciones sanas y con OATM en modelos 3D tomados de CBCT
Compararon 20 sujetos clasificados en el grupo III según RDC/TMD y 40 controles asintomáticos
Paniagua y Analizar los cambios de forma en cols, 2011 reabsorción ósea en pacientes con OATM, en modelo 3D tomados de CBCT
Estudio longitudinal con 26 sujetos con OATM, con indicación se tratamiento ortodóncico-quirúrgico. El análisis de forma se realiza:

Pretratamiento

Posterior al uso de plano

Al año post cirugía

A 2 años posterior a cirugía

Anjos Pontual Determinar la prevalencia de cambios y cols,2012 óseos degenerativos y movilidad de las ATMs en CBCT
Realizaron un estudio transversal con 319 sujetos para la evaluación imagenológica de ambas ATMs, analizando aplanamiento, erosión, osteofitos, esclerosis y pseudoquistes.
Koos y cols, Evaluar cambios degenerativos e 2013 inflamatorios articulares comparando pacientes sanos y pacientes con artritis idiopática juvenil observados en CBCTy RM con contraste
Estudio transversal. 46 sujetos, 23 con artritis idiopática juvenil y 23 sanos Se evaluó el grado de destrucción articular (CBCT) e inflamación (RM). Las articulaciones fueron clasificadas en clases: $A$, sin cambios, $B$ deformación y $C$ destrucción. Además fueron subclasificadas según grado: I aplanamiento, II erosión y III osteofitos, y la severidad de cada grado en 1, 2 y 3 .
Se observaron cambios en estructura ósea condilar en un $63,7 \%$ de las articulaciones observadas. De las afectadas, según clasificación: tipo $\mathrm{F} 8 \%$, tipo E $19 \%$, tipo D $19 \%$ y tipo S $14 \%$. De las articulaciones con seguimiento, se observó una tendencia a la progresión degenerativa, aumentando la proporción de tipo $D$ y $E$.

La morfología fue estadísticamente diferentes, los sujetos con OATM presentan medidas menores en promedio: $1.9 \mathrm{~mm}$ en la derecha y $2.3 \mathrm{~mm}$ en la izquierda, exhibiendo reabsorción anterior en el polo lateral

Tanto para el cóndilo derecho como en el izquierdo presentaron cambios remodelativos leves en el polo superior, al retiro del plano. Cambios regresivos moderados en el polo anterior y superior al año postcirugía y cambios severos a los dos años postcirugía comparados con el modelo inicial precirugía.

No se encontró diferencia estadísticamente significativa entre ambas ATMs. Los cambios degenerativos son mas prevalentes en mujeres $y$ aumentan con la edad. No existe correlación entre grado de movilidad articular y cambios degenerativos.

En el grupo control, un $21 \%$ de las articulaciones presentaron cambios estructurales leves. Los cambios estructurales observados se concentraron principalmente en clases leves. En el grupo con artritis, un $83 \%$ de las articulares presentaron cambios estructurales avanzados, con mayor concentración en fases severas. No se observó una correlación estadísticamente significativa con edad ni sexo.

En pacientes con asimetría mandibular la morfología 3D condílea en el lado desviado difería del lado no desviado, indicando una asociación entre la función mandibular asimétrica y la remodelación articular. La incongruencia en el lado desviado resultó en una reducción de áreas de contacto y aumento en la tensión, con cambios en densidad ósea.

Se encontraron diferencias estadísticamente significativas entre pacientes con EDATM y los controles, consistentemente localizadas en los polos medial y lateral. Concluyen que el registro condilar interindividual y las evaluaciones longitudinales son confiable y pueden usarse para cuantificar diferencias óseas sutiles en la morfología condilar tridimensional. 
Tabla 1. Resumen descriptivo de los papers seleccionados $(n=18)$ (continuación)

\begin{tabular}{|c|c|c|c|}
\hline Autor y año & Objetivo & Metodología & Conclusiones \\
\hline $\begin{array}{l}\text { Schiffman } \\
\text { cols, } 2014\end{array}$ & $\begin{array}{l}\text { Revisar y realizar recomendaciones } \\
\text { consensuadas, para la determinación de } \\
\text { nuevos algoritmos de diagnóstico del Eje } \\
\text { I y nuevos instrumentos del Eje II para la } \\
\text { clínica e investigación en TTM }\end{array}$ & $\begin{array}{l}\text { Se realizaron talleres y simposios } \\
\text { de revisión y consenso mediante un } \\
\text { proceso estructurado formal entre } \\
\text { miembros del "International RDC/TMD } \\
\text { Consortium Network" y "Orofacial Pain } \\
\text { Special Interest Group" y se genera un } \\
\text { nuevo protocolo de DC/TMD basado en } \\
\text { evidencia. }\end{array}$ & $\begin{array}{l}\text { Concluyen que nuevo protocolo DC/TMD } \\
\text { es apropiado para su uso en entornos } \\
\text { clínicos y de investigación puesto que } \\
\text { el uso de instrumentos más completos } \\
\text { aumentan la detección de patologías } \\
\text { en los Ejes I y II. Los instrumentos } \\
\text { validados permiten la identificación de } \\
\text { pacientes con una mayor variedad de } \\
\text { presentaciones en TTM tanto las simples } \\
\text { como las más complejas. }\end{array}$ \\
\hline
\end{tabular}

Larheim y Realizar una actualización de la Revisión de la literatura.

cols, 2015 imagenología de ATM usando CBCT

EI CBCT en la evaluación de ATM constituye una alternativa rentable y dosis efectiva a la TC, puede ser más sensible a artefactos de movimiento sin embargo es superior a la radiología convencional y a la RM en la evaluación de anormalidades óseas, para evaluación de actividad inflamatoria y anomalías de tejidos blandos la RM es el método de elección.

Cevidanes y Investigar las variaciones morfológicas cols, 2015 de cóndilos de sujetos con EDATM por medio de superposición de imágenes de modelos 3D tomados en CBCT
Compararon 3 grupos de pacientes: 69 sujetos con larga data de EDATM, 15 sujetos con diagnóstico inicial de OATM y 7 sujetos sanos.
El test DiPromPerm utilizado por los autores permiten evaluar a través de biomarcadores de imagen, los cóndilos de manera cuantitativa, permitiendo clasificaciones las EDATM de manera estadística.

Observaron que los pacientes con EDATM tienen medidas de volumen condilar en promedio menores que los sujetos sanos, excepto en el polo anterior.

Gomes y Evaluar en variaciones morfológicas de cols, 2015 OATM con un enfoque estadístico de las imágenes volumétricas a partir de CBCT

Estudio transversal con 7 sujetos sanos, 15 sujetos con OATM inicial y 69 sujetos con OATM de larga data. Se generaron modelos 3D de cada articulación. Se utilizó el software SPHARM-PDM Se calcularon los patrones de variación entre los cóndilos de los 3 grupos, indicando áreas de reabsorción, de proliferación y/o sin cambios. Se realizó un agrupamiento jerárquico basados en la similaridad morfológica condilar.

Los cóndilos con OATM tuvieron en promedio menor tamaño en todas las dimensiones, especialmente en zona superior, y excepto en superficie anterior y polo medial donde se observó aposición reparativa. En los cóndilos con AOTM inicial se observó un marcado aplanamiento en el polo lateral condilar. Los sujetos con OATM de evolución prolongada presentan cambios morfológicos marcados

Se provee un índice diagnóstico preeliminar de cambios osteoatríticos 3D en la morfología condilar mandibular, que podría ser un primer paso para un diagnóstico más certero de esta condición.

Compararon 32 sujetos asimétricos y 34 sujetos simétricos como grupo control
Los volúmenes tanto del cóndilo como de la fosa y del espacio articular del lado más pequeño fueron estadísticamente significativos en el grupo de estudio. Señalan que para evaluar asimetría hay que considerar las características de la fosa, la altura, el ancho y la posición del cóndilo.

Para la mayoría de los pacientes estudiados, la artrocentesis ofreció resultados favorables a largo plazo. La severidad de los hallazgos clínicos y radiológicos preoperatorios no predice el éxito de la artrocentesis. La falta de correlación hallazgos clínicos-radiológicos niega el uso de la clasificación de Wilkes quien establece que la severidad de signos clínicos van correlacionados con los radiológicos 
Tabla 1. Resumen descriptivo de los papers seleccionados ( $n=18)$ (continuación)

\begin{tabular}{|c|c|c|c|}
\hline Autor y año & Objetivo & Metodología & Conclusiones \\
\hline $\begin{array}{l}\text { Tamimi } \\
\text { cols, } 2018\end{array}$ & $\begin{array}{l}\text { Analizar la anatomía, función, crecimiento } \\
\text { y desarrollo de la ATM y cómo los } \\
\text { cambios en el crecimiento pueden afectar } \\
\text { la morfología craneofacial. }\end{array}$ & $\begin{array}{l}\text { Revisión de la literatura. } \\
\text { Se muestran y analizan imágenes en } \\
\text { CBCT y RM. }\end{array}$ & $\begin{array}{l}\text { La ATM es una estructura compleja } \\
\text { relacionada mental y funcionalmente con } \\
\text { el resto del complejo craneomandibular. } \\
\text { La clave de la correcta interpretación } \\
\text { imagenológica de la ATM reside en un } \\
\text { conocimiento y comprensión exhaustivo } \\
\text { de su anatomía y de su función/ } \\
\text { disfunción. }\end{array}$ \\
\hline
\end{tabular}

\begin{tabular}{ll}
\hline $\begin{array}{l}\text { Hilgenberg- } \\
\text { Sydney } \\
\text { cols, } 2018\end{array}$ & $\begin{array}{l}\text { Evaluar la validez del diagnóstico } \\
\text { imagenológico en TC y CBCT en } \\
\text { pacientes con EDA comparados con } \\
\text { criterios clínicos }\end{array}$ \\
\hline
\end{tabular}

Ribera y cols, Desarrollar un análisis de variabilidad 2019 de la forma que permita estadiar los cambios óseos en EDATM, lo llamaron "Deep learning neural network"

Gumussoy y Evaluar la utilidad del análisis óseo Duman, 2019 microestructural 3D en pacientes con EDATM
Revisión sistemática

Tomaron una muestra de 259 CBCT y sometieron al test a 34 modelos $3 \mathrm{D}$, los cuales fueron clasificado en 6 grupos

Seleccionaron 147 CBCT de ATM en 88 pacientes y los agruparon en tres grupos de acuerdo a la clasificación de MUI en 1990.

Grupo 0: control

Grupo 1: cambios erosivos leves

Grupo 2: cambios erosivos severos.
Concluyen que eo CBCT es válida para evaluar la progresión en el tiempo de la EDA, sin embargo no debe usarse como examen de rutina en pacientes sanos.

Concluyeron que utilizar "Deep learning neural network"es el método mas preciso para clasificar la EDATM en base a metodología 3D

La dimensión fractal 3D en cada CBCT condilar y arrojó un índice más bajo junto con la observación de cambios más severos en el grupo 2. Siendo las diferencias estadísticamente significativas para cada grupo. Este estudio preliminar puede ser de utilidad para mejorar la capacidad diagnóstica del cone beam en pacientes con EDATM

TTM, Trastornos Temporomandibulares, ATM, Articulación Temporomandibular; OATMD, Osteoartritis Temporomandibular; EDATM, Enfermedad Degenerativa Articular Temporomandibular; TC, Tomografia Computarizada; CBCT, Cone beam Computer Tomography; RM, Resonancia Magnética; RDC/TMD, Research diagnostic criteria/ Temporomandibular disordes; DC/TMD, diagnostic criteria/Temporomandibular disordes; SPHARM-PDM, The 3-D shape correspondence analysis

\section{Bibliografía}

1. Lin H, Zhu P, Lin Y, Wan S, Shu X, Xu Y, et al. Mandibular asymmetry: a threedimensional quantification of bilateral condyles. Head Face Med. 2013;9:42. 2. Tamimi D, Jalali E, Hatcher D. Temporomandibular joint imaging. Ra-diol Clin North Am. 2018:56:157-175.

3. Wang L, Lazebnik M, Detamore MS. Hyaline cartilage cells outperform mandibular condylar cartilage cells in a TMJ fibrocartilage tissue engi-neering application. Osteoarthritis cartilage. 2009;17:346-53.

4. Schilling J, Gomes LCR, Benavides E, Nguyen T, Paniagua B, Styner M, et al. Regional 3D superimposition to assess temporomandibular joint condylar morphology. Dentomaxillofac Radiol. 2014;43:20130273.

5. Wang X, Zhang J, Gan Y, Zhou Y. Current understanding of pathogen-esis and treatment of TMJ osteoarthritis. J Dent Res. 2015;94:666-73.

6. Nitzan DW, Svidovsky J, Zini A, Zadik Y. The effect of arthrocentesis on symptomatic osteoarthritis of the temporomandibular joint and analysis of the effect of preoperative clinical and radiologic features. J Oral Max-illofac Surg. 2017;75:260267.

7. Schiffman E, Ohrbach R, Truelove E, et al. Diagnostic criteria for temporomandibular disorders (DC/TMD) for clinical and research applica-tions: recommendations of the International RDC/TMD Consortium Network* and orofacial pain special interest group. J Oral Facial Pain Headache 2014;28:6-27.

8. Larheim TA, Abrahamsson AK, Kristensen M, Arvidsson LZ. Temporo-mandibular joint diagnostics using CBCT. Dentomaxillofac Radiol. 2015:44:20140235.

9. Ahmad M, Hollender L, Anderson Q, Kartha K, Ohrbach R, Truelove EL, et al. Research diagnostic criteria for temporomandibular disorders (RDC/TMD): development of image analysis criteria and examiner reli-ability for image analysis. Oral Surg Oral Med Oral Pathol Oral Radiol Endod. 2009;107:844-60.

10. Dos Anjos Pontual ML, Freire JS, Barbosa JM, Frazão MA, Dos Anjos Pontual A. Evaluation of bone changes in the temporomandibular joint using cone beam CT. Dentomaxillofac Radiol. 2012; 41:24-29.

11. Farah CS, Balasubramaniam R, McCullough MJ. Contemporary oral medicine. Switzerland: Springer; 2016.

12. Koyama JI, Nishiyama H, Hayashi T. Follow-up study of condylar bony changes using helical computed tomography in patients with tem-poromandibular disorder. Dentomaxillofac Radiol. 2007;36:472-477.

13. Hilgenberg-Sydney PB, Bonotto DV, Stechman-Neto J, Zwir LF, PachecoPereira C, Canto GL et al. Diagnostic validity of $\mathrm{CT}$ to assess degenerative temporomandibular joint disease: A systematic review. Dentomaxillofac Radiol. 2018; 47:20170389.
14. Tanaka E, Detamore MS, Mercuri LG. Degenerative disorders of the temporomandibular joint: etiology, diagnosis, and treatment. J Dent Res. 2008;87:296-307.

15. Koos B, Tzaribachev N, Bott S, Ciesielski R, Godt A. Classification of temporomandibular joint erosion, arthritis, and inflammation in patients with juvenile idiopathic arthritis. J Orofac Orthop. 2013;74:506-19.

16. Gomes LR, Gomes M, Jung B, Paniagua B, Ruellas AC, Gonçalves JR, et al. Diagnostic index of three-dimensional osteoarthritic changes in temporomandibular joint condylar morphology. J Med Imaging (Bellingham). 2015; 2:034501-9.

17. Uemura S, Nakamura M, Iwasaki H, Fuchihata H. A roentgenological study on temporomandibular joint disorders. Morphological changes of TMJ in arthrosis. Dent Radiol. 1979;19:224-237.

18. Ahmad M, Schiffman EL. Temporomandibular joint disorders, and oro-facial pain. Dent Clin North Am. 2016;60:105-24.

19. Cevidanes LH, Walker DG, Styner M, Lim PF. Condylar resorption in patients with TMD. Craniofacial Growth Ser. 2009;46:147-157.

20. Paniagua B, Cevidanes L, Walker D, Zhu H, Guo R, Styner M. Clinical application of SPHARM-PDM to quantify temporomandibular joint os-teoarthritis. Comput Med Imaging Graph. 2011;35:345-52.

21. Kim JY, Kim BJ, Park KH, Huh JK. Comparison of volume and position of the temporomandibular joint structures in patients with mandibular asymmetry. Oral Surg Oral Med Oral Pathol Oral Radiol. 2016:122:772-780.

22. Habets LL, Bezuur JN, Naeiji M, Hansson TL. The orthopantomogram, an aid in diagnosis of temporomandibular joint problems. II. The verti-cal symmetry. J Oral Rehabil. 1988;15:465-71.

23. Cevidanes LHS, Gomes LR, Jung BT, Gomes MR, Ruellas ACO, Gon-calves $J R$, et al. 3D superimposition and understanding temporoman-dibular joint arthritis. Orthod Craniofacial Res. 2015;18:18-28.

24. Gumussoy I, Duman SB. Alternative cone-beam CT method for the analysis of mandibular condylar bone in patients with degenerative joint disease. Oral Radiol. 2019; 29:1-6.

25. Muir CB, Goss, AN. The radiologic morphology of asymptomatic temporomandibular joints. Oral Surgery, Oral Medicine, Oral Pathology. 1990; 70:34954.

26. Ribera NT, de Dumast P, Yatabe M, Ruellas A, loshida M, Paniagua B, et al. Shape variation analyzer: a classifier for temporomandibular joint damaged by osteoarthritis. Proc SPIE Int Soc Opt Eng. 2019;10950:1095021. 